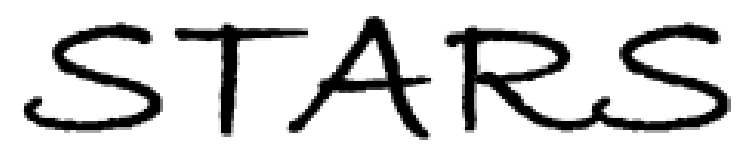

University of Central Florida

STARS

Faculty Bibliography 2010s

Faculty Bibliography

$1-1-2012$

\title{
Dynamic response of a polymer-stabilized blue-phase liquid crystal
}

Jin Yan

University of Central Florida

Yuan Chen

University of Central Florida

Shin-Tson Wu

University of Central Florida

Shih-Hsien Liu

Kung-Lung Cheng

See next page for additional authors

Find similar works at: https://stars.library.ucf.edu/facultybib2010

University of Central Florida Libraries http://library.ucf.edu

This Article is brought to you for free and open access by the Faculty Bibliography at STARS. It has been accepted for inclusion in Faculty Bibliography 2010 s by an authorized administrator of STARS. For more information, please contactSTARS@ucf.edu.

\section{Recommended Citation}

Yan, Jin; Chen, Yuan; Wu, Shin-Tson; Liu, Shih-Hsien; Cheng, Kung-Lung; and Shiu, Jyh-Wen, "Dynamic response of a polymer-stabilized blue-phase liquid crystal" (2012). Faculty Bibliography 2010s. 3525. https://stars.library.ucf.edu/facultybib2010/3525

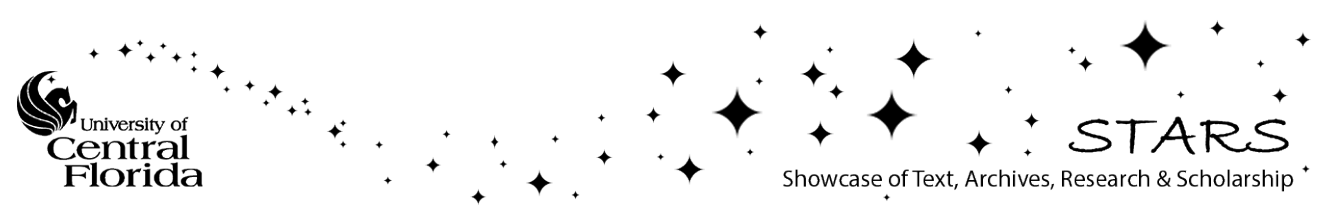


Authors

Jin Yan, Yuan Chen, Shin-Tson Wu, Shih-Hsien Liu, Kung-Lung Cheng, and Jyh-Wen Shiu

This article is available at STARS: https://stars.library.ucf.edu/facultybib2010/3525 


\section{Dynamic response of a polymer-stabilized blue-phase liquid crystal}

Cite as: J. Appl. Phys. 111, 063103 (2012); https://doi.org/10.1063/1.3694733

Submitted: 06 February 2012 . Accepted: 07 February 2012 . Published Online: 21 March 2012

Jin Yan, Yuan Chen, Shin-Tson Wu, Shih-Hsien Liu, Kung-Lung Cheng, and Jyh-Wen Shiu

\section{ARTICLES YOU MAY BE INTERESTED IN}

Electro-optic response of polymer-stabilized blue phase liquid crystals

Applied Physics Letters 105, 011119 (2014); https://doi.org/10.1063/1.4890031

A full-color reflective display using polymer-stabilized blue phase liquid crystal

Applied Physics Letters 102, 081102 (2013); https://doi.org/10.1063/1.4793750

A large Kerr constant polymer-stabilized blue phase liquid crystal

Applied Physics Letters 98, 081109 (2011); https://doi.org/10.1063/1.3559614

\section{Applied Physics Reviews} Now accepting original research

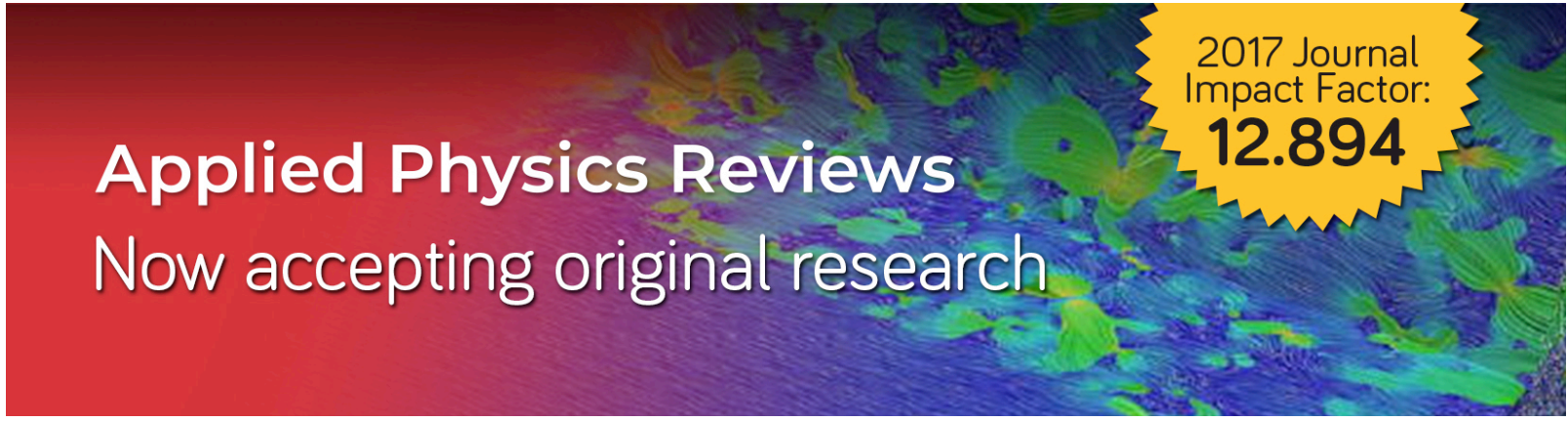




\title{
Dynamic response of a polymer-stabilized blue-phase liquid crystal
}

\author{
Jin Yan, ${ }^{1}$ Yuan Chen, ${ }^{1}$ Shin-Tson Wu, ${ }^{1, a)}$ Shih-Hsien Liu, ${ }^{2}$ Kung-Lung Cheng, ${ }^{2}$ \\ and Jyh-Wen Shiu ${ }^{3}$ \\ ${ }^{1}$ College of Optics and Photonics, University of Central Florida, Orlando, Florida 32816, USA \\ ${ }^{2}$ Material and Chemical Research Laboratories, Industrial Technology Research Institute, Hsinchu, Taiwan \\ ${ }^{3}$ Display Technology Center, Industrial Technology Research Institute, Hsinchu, Taiwan
}

(Received 6 February 2012; accepted 7 February 2012; published online 21 March 2012)

\begin{abstract}
Fast response time is the most attractive feature of polymer-stabilized blue phase liquid crystals (PS-BPLCs). We have investigated the dynamic response of a PS-BPLC under various electric fields and found that the response time becomes slower as the applied electric field exceeds a critical field. Further analyses of experimental data reveal that two relaxation processes are involved. Possible mechanism is proposed to explain the behavior of each process. These results provide useful guidelines for achieving fast response time without hysteresis. (C) 2012 American Institute of Physics. [http://dx.doi.org/10.1063/1.3694733]
\end{abstract}

\section{INTRODUCTION}

Polymer-stabilized blue phase liquid crystal (PS-BPLC) ${ }^{1-3}$ is emerging as a promising candidate for display ${ }^{4-7}$ and photonic $^{8,9}$ applications because it exhibits some attractive properties, such as alignment-layer free, submillisecond response time, ${ }^{10}$ and optically isotropic dark state. Among these features, fast response time is the most desirable one because it enables color sequential displays using RGB LEDs, which in turn eliminates the spatial color filters. Consequently, the optical efficiency and resolution density are all tripled. Recently, it was found that the response time of a PS-BPLC could vary from microseconds to several milliseconds, ${ }^{11,12}$ which is not only related to the viscosity of the host LC materials, but also related to the strength and distribution of electric fields. ${ }^{13}$ Therefore, there is an urgent need to understand the dynamic response behavior of a BPLC device in order to keep fast response time.

In this paper, we investigated the dynamic response behaviors of a PS-BPLC composite under different electric fields. We focus on the phase decay response instead of transmittance, ${ }^{10-12}$ because the latter usually smears the underlying physics of the dynamic response due to the sinusoidal modulation. It is found that there is a critical field, which not only determines the onset of hysteresis but also plays an important role to the response time of a PS-BPLC composite.

\section{EXPERIMENT}

The BPLC host employed in this study consists of 94.11 wt. \% JM2069-145 (ITRI, Taiwan) and 5.89 wt. \% of a high helical twisting power chiral dopant $(\mathrm{HTP} \approx 100 / \mu \mathrm{m}$, Formula I-2 in Ref. 14). The nematic LC host JM2069-145 has the following physical properties: $\Delta n=0.215$ at $\lambda=633$ $\mathrm{nm}, \Delta \varepsilon=50$ at $1 \mathrm{kHz}$ and $21^{\circ} \mathrm{C}$, and clearing temperature $T_{c}$ $=80^{\circ} \mathrm{C}$. The BPLC host is then mixed with $10 \mathrm{wt}$. $\%$ of photocurable monomers [4 wt. \% TMPTA (1,1,1-Trimethylolpropane Triacrylate, Sigma Aldrich) and 6 wt. \% RM257,

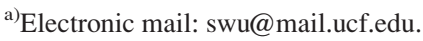

Merck], as well as a small amount of photoinitiator $(\sim 0.65$ wt. \%) to form a precursor. The transition temperature between chiral nematic and blue phase is $43.5^{\circ} \mathrm{C}$ during heating process and $38.6^{\circ} \mathrm{C}$ during cooling process. To investigate the dynamic response of the BPLC composite, a uniform electric field is preferred. Therefore, we injected the precursor to a vertical field switching (VFS) cell. ${ }^{13}$ The VFS cell is made of two ITO glass substrates and the cell gap is $5.15 \mu \mathrm{m}$. We controlled the temperature of the cell at $43.5^{\circ} \mathrm{C}$ during the cooling process and irradiated UV light with an intensity of $2 \mathrm{~mW} / \mathrm{cm}^{2}$ for $30 \mathrm{~min}$. After UV exposure, a self-assembled BPLC composite was stabilized.

Figure 1 depicts the measurement setup. The absorption axes of polarizer and analyzer are at $45^{\circ}$ and $-45^{\circ}$ azimuthal angles with respect to the incident plane, respectively. For a normally incident light, there is no phase retardation under an electric field because the induced optic axis is along the electric field direction which is parallel to the light propagation direction, like a C-plate. Therefore, to accumulate phase retardation, the incident light should be set at an oblique angle. In general, the phase retardation for a uniaxial medium at oblique incidence can be expressed as:

$$
\varphi(E)=\left(k_{e, z}-k_{o, z}\right) d,
$$

where $d$ is the cell gap, and $k_{o, z}$ and $k_{e, z}$ are the normal components of wavevectors of ordinary and extraordinary waves, respectively. The cell was immersed in a glycerol container. Because of the matched refractive index between the glycerol liquid and BP cell, the light can propagate inside the cell with a very large angle to accumulate more phase retardation. Under such a circumstance, the phase retardation can be further derived from Eq. (1) as: ${ }^{15}$

$$
\varphi(E)=\frac{2 \pi}{\lambda} n_{o}(E) d\left[\sqrt{1-\frac{n_{g}^{2} \sin ^{2} \theta}{n_{e}^{2}(E)}}-\sqrt{1-\frac{n_{g}^{2} \sin ^{2} \theta}{n_{o}^{2}(E)}}\right]
$$

where $\lambda$ is the laser wavelength, $n_{g}$ is the refractive index of glycerol liquid and $\theta$ is the incident angle in the glycerol. 


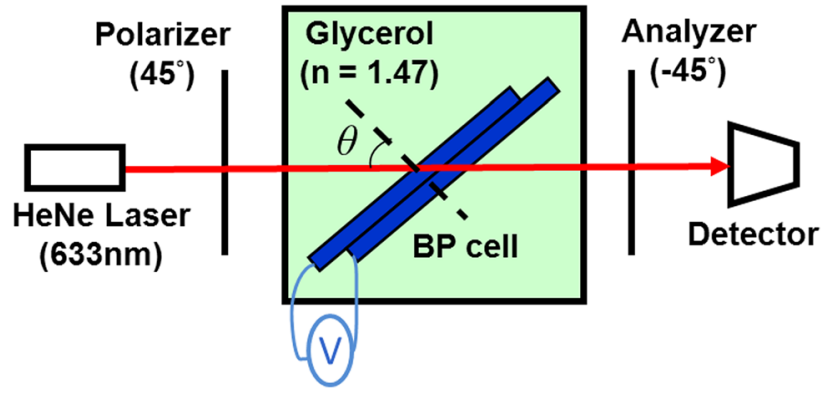

FIG. 1. (Color online) Experimental setup for electro-optic measurement of the prepared VFS cell.

$n_{o}(E)$ and $n_{e}(E)$ are electric field dependent ordinary and extraordinary refractive indices of the PS-BPLC composite, which can be described as: ${ }^{16,17}$

$$
\begin{gathered}
n_{o}(E)=n_{i}-\Delta n_{\text {ind }}(E) / 3, \\
n_{e}(E)=n_{i}+2 \Delta n_{\text {ind }}(E) / 3,
\end{gathered}
$$

with

$$
\Delta n_{\text {ind }}=\Delta n_{s}\left(1-\exp \left[-\left(E / E_{s}\right)^{2}\right]\right) .
$$

In Eq. (5), $\Delta n_{s}$ is the saturated induced birefringence and $E_{s}$ is the saturated electric field. From Eqs. (2)-(5), a larger incident angle leads to larger phase retardation. ${ }^{17}$ In our experiment, we chose $\theta=70^{\circ}$. A $1-\mathrm{kHz}$ square-wave AC signal was applied to drive the BPLC cell. The detector was connected to a LabVIEW data acquisition system and oscilloscope to record the voltage and time dependent transmittance curves.

\section{RESULTS}

Figure 2(a) depicts the electric field dependent phase retardation. The transmittance is related to the phase retardation as:

$$
T=\sin ^{2}(\varphi / 2)
$$

Here we converted the transmittance to phase retardation. The small light leakage at voltage-off state is due to the stress birefringence of the glass substrates employed. This light leakage is not of our concern in this specific study since we focus on the dynamic response of the PS-BPLC sample. Through fitting with Eqs. (2)-(5), we obtained $\Delta n_{s}=0.12$ and $E_{s}=12.8 \mathrm{~V} / \mu \mathrm{m}$, the Kerr constant $K=\Delta n_{s} /\left(\lambda E_{s}^{2}\right)=1.2 \mathrm{~nm} / \mathrm{V}^{2}$. From Fig. 2(a), it is interesting to notice that the hysteresis starts to appear when $E>8.5 \mathrm{~V} / \mu \mathrm{m}$, which we call critical field $\left(E_{c}\right)$. Semiempirically, we found that the critical field is related to the Kerr constant of the BPLC material as:

$$
E_{c} \propto 1 / \sqrt{K}
$$

For instance, the Kerr constant $K_{1}$ here is $\sim 1.2 \mathrm{~nm} / \mathrm{V}^{2}$ and the critical field $E_{c l} \sim 8.5 \mathrm{~V} / \mu \mathrm{m}$; while in Ref. 18 the Kerr constant $K_{2} \sim 7.5 \mathrm{~nm} / \mathrm{V}^{2}$ and the critical field $E_{c 2} \sim 3.5 \mathrm{~V} / \mu \mathrm{m}$. The ratio between $E_{c 1}$ and $E_{c 2}$ is $\sim 2.4$ and $\sqrt{K_{2} / K_{1}} \sim 2.5$. The agreement is reasonably good although the two BPLC material systems are quite different. Since the operation voltage of a BPLC system is proportional to $1 / \sqrt{K}$ (Ref. 6), it is
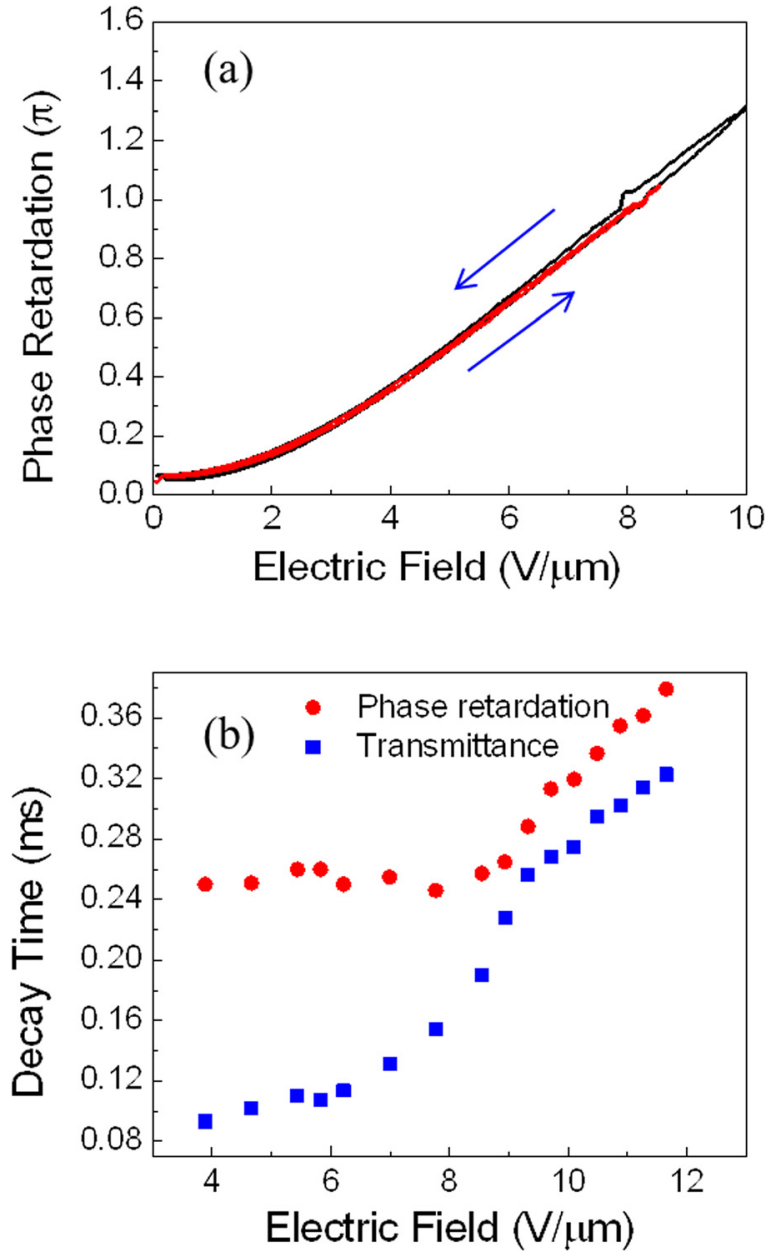

FIG. 2. (Color online) (a) Electric field dependent phase retardation and hysteresis; (b) phase retardation and transmittance decay time $(100 \%-10 \%)$ when different electric fields are removed.

considered that the critical field is also related to the liquid crystal orientation and, as a result, the unwinding of the helical twist. A BPLC with a larger Kerr constant is easier to be unwound, which corresponds to a smaller critical field.

To measure response time, we let the PS-BPLC sample relax from different applied voltages and recorded the dynamic relaxation process by a digital oscilloscope. The measured amplitude relaxation was also converted to phase relaxation according to Eq. (6). Figure 2(b) shows the amplitude and phase decay times from $100 \%$ to $10 \%$ of the modulation depth. It is found the phase decay time is slower than the amplitude decay time, especially when the phase retardation is less than $1 \pi$. This is because of the sinusoidal modulation as Eq. (6) shows. Although amplitude response time is commonly used for display applications, phase retardation response time can better reveal the underlying physical mechanism. From Fig. 2(b), it is found that the phase decay time is almost a constant when the applied field is below $8.5 \mathrm{~V} / \mu \mathrm{m}$, but starts to increase as $E>E_{c}$.

To further investigate the dynamic response of the PSBPLC sample, we fitted the phase decay curves with a double relaxation equation:

$$
\varphi(t)=A_{1} e^{-t / \tau_{1}}+A_{2} e^{-t / \tau_{2}}+\varphi_{0} .
$$



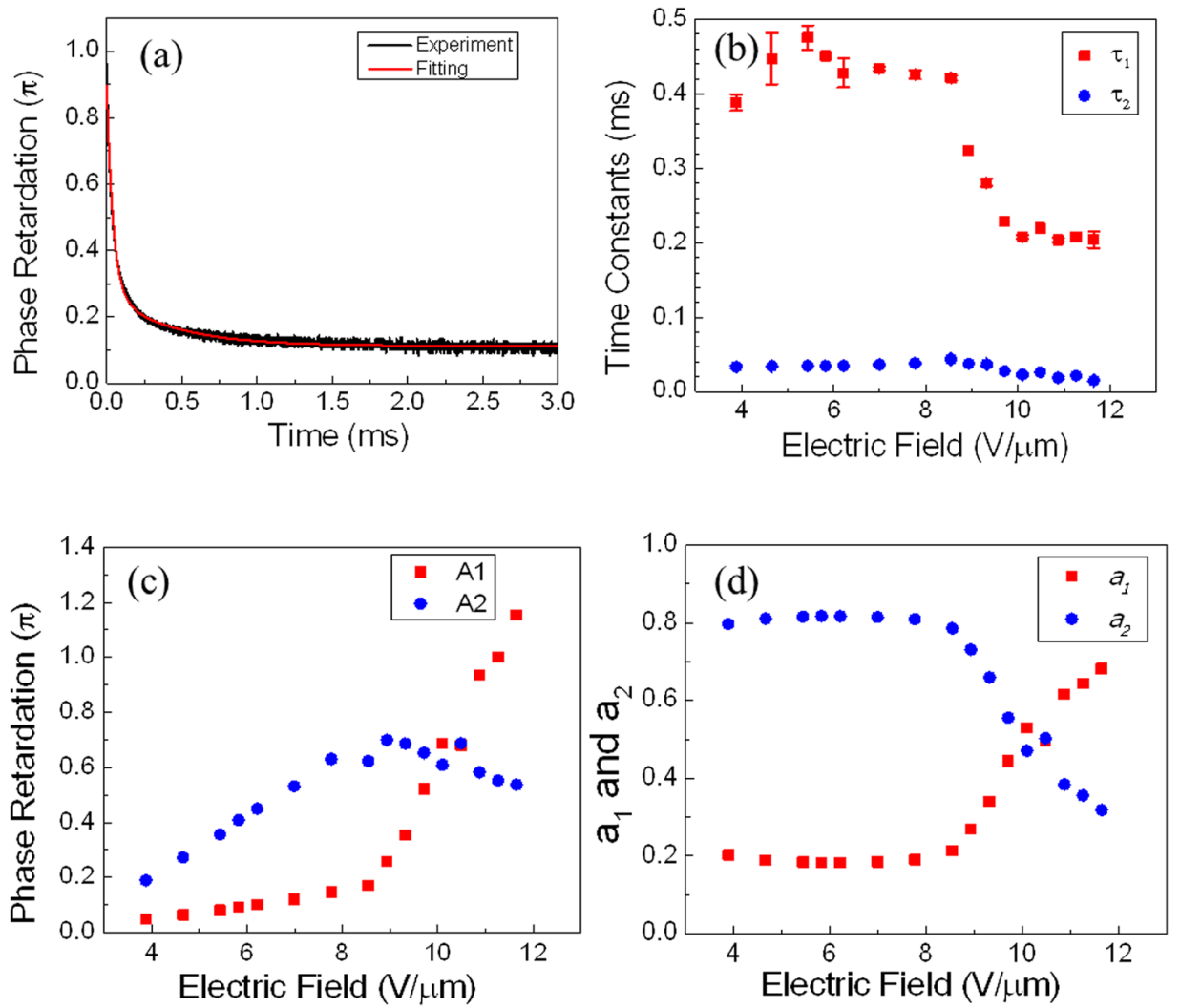

FIG. 3. (Color online) (a) Transient decay process at $44 \mathrm{~V}$; (b) decay time constants $\tau_{1}$ and $\tau_{2}$ when different electric fields are removed; (c) phase retardation amplitude $A_{1}$ and $A_{2}$ under different electric fields; and (d) normalized phase retardation amplitude $a_{1}$ and $a_{2}$ for different electric fields.
The first term in Eq. (8) represents slow process and the second term represents fast process. $\tau_{1}$ and $\tau_{2}$ are the averaged time constants, and $A_{1}$ and $A_{2}$ are the phase retardations contributed by the slow and fast processes, respectively. $\varphi_{0}$ is the phase retardation caused by the initial light leakage and residual birefringence. The reason that we use the double relaxation equation will be explained in more details later. Figure 3(a) shows the measured phase decay process and the fitting using Eq. (8) when a voltage of $44 \mathrm{~V}$ is removed at $t=0$. As shown in Fig. 3(a), the fitting with Eq. (8) is quite good.

For other applied fields, we also fitted the experimental curves with Eq. (8). The values of $\tau_{1}, \tau_{2}, A_{1}$, and $A_{2}$ for different electric fields are plotted in Figs. 3(b) and 3(c). It is interesting to note that $\tau_{2}$ remains constant for all the applied electric fields but $\tau_{1}$ starts to decrease when $E>8.5 \mathrm{~V} / \mu \mathrm{m}$, which is the critical field for hysteresis to occur. In Fig. 3(c), the phase retardations for both fast and slow processes first increase linearly with $E$, but at $\sim 8.5 \mathrm{~V} / \mu \mathrm{m}$ there is a transition point, after which $A_{1}$ starts to increase more quickly while $A_{2}$ decreases. We also plotted the contributions of $A_{1}$ and $A_{2}$ to the overall phase retardation in Fig. 3(d). Here we define $a_{1}=A_{1} /\left(A_{1}+A_{2}\right)$ and $a_{2}=A_{2} /\left(A_{1}+A_{2}\right)$. Below $E_{c}, a_{1}$ and $a_{2}$ remain constants but $a_{1}$ starts to increase when $E>E_{c}$ while $a_{2}$ starts to decrease, which means the slow process makes more contribution to the overall phase retardation.

\section{DISCUSSION}

We proposed the following physical model to qualitatively explain the data in Fig. 3. For a nematic LC with a fixed pretilt angle, the phase decay process can be described by a single exponential decay under small angle approximation. ${ }^{19,20}$ However, in blue phases, similar to a polymerdispersed liquid crystal (PDLC) system, except the LC directors are arranged in a double-twisted structure, the pretilt angles are distributed in all the directions. Without losing generality, we draw a schematic diagram to represent the randomly distributed LC directors surrounded by polymer network, as shown in Fig. 4(a). The LC directors have different pretilt angles with respect to the applied electric field. Therefore, they will rotate by different angles under an electric field. Those who rotate by a larger angle will have slower response time. Moreover, the anchoring force provided by the polymer network is not uniform over the entire LC medium. For example, the LC directors close to the polymer network experience a stronger anchoring force while those in the center experience a weaker force. A stronger anchoring force helps restore the LCs with faster response time. As a result, the responses of LC directors are different and there are multiple decay processes going on. Here, we take an average effect and approximate the decay process as a double relaxation process.

When the electric field is weak, the LC directors only rotate by a relatively small angle. Let us assume there are multiple time constants with $\tau_{a}<\tau_{b}<\tau_{c}<\tau_{d}<\tau_{e}$. The LCs with small pretilt angle and strong anchoring force have fastest response time, while those with large pretilt angle and weak anchoring force have slowest response time. The overall effect is approximated by a double relaxation process, with $\tau_{2}$ representing the averaged effects of $\tau_{a}$ to $\tau_{d}$, and $\tau_{1}$ representing the slow response time $\tau_{e}$, as shown in Fig. 4(b). As the electric field increases but below the critical field, the LC rotation angles increase, but still within a 
(a)

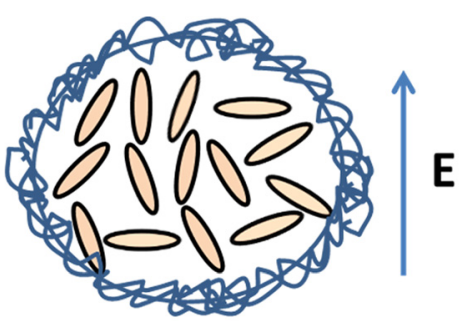

(b)

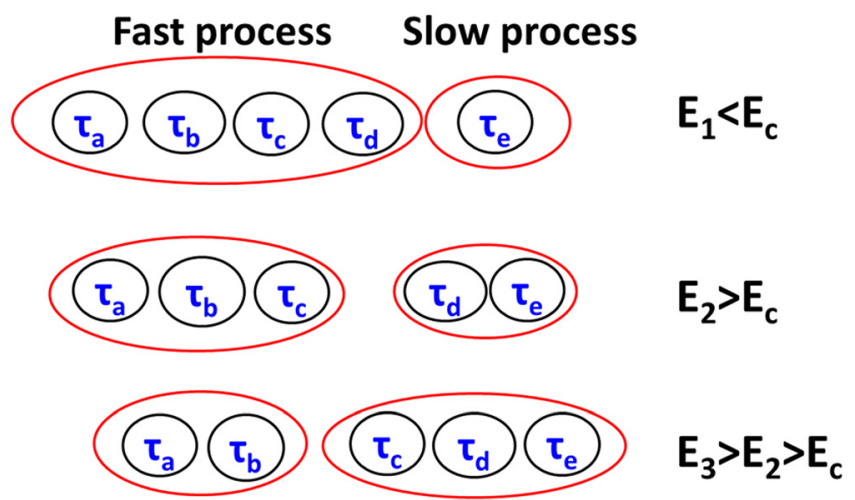

FIG. 4. (Color online) (a) Schematic diagram of the liquid crystals surrounded by polymer network and (b) schematic diagram of how fast and slow processes change with the applied electric field.

certain limit. Therefore, the time constants and the ratio between $A_{1}$ and $A_{2}$ are insensitive to the applied electric field. However, when $E>E_{c}$ some LC directors have been reoriented by a large angle which is above the critical angle and causes unwinding of helical twist. As a result, the LC relaxation process does not follow the same route as the rising path, which leads to hysteresis. Similarly, the large rotation angle also leads to a significant change in the dynamic decay process. For example, $\tau_{c}$ and $\tau_{d}$ become much slower so that they join $\tau_{e}$ to contribute to the slow process. But they are still faster than $\tau_{e}$ so the averaged time constant $\tau_{1}$ decreases as $E>E_{c}$. As to the fast process, $A_{2}$ decreases because LCs with time constants $\tau_{c}$ and $\tau_{d}$ join the slow process and only those LCs with time constants $\tau_{a}$ and $\tau_{b}$ make contribution to the phase retardation of the fast process. Moreover, since $\tau_{a}$ and $\tau_{b}$ are faster than $\tau_{c}$ and $\tau_{d}$, the averaged time constant $\tau_{2}$ also slightly decreases.

The response time information plays an important role to device design and optimization. From our results, the response time becomes slower and the hysteresis starts to appear as the electric field exceeds the critical field. Therefore, it is very important to keep the maximum electric field below this critical field in order to achieve fast response time and hysteresis-free performance. A thicker cell gap helps reduce the peak electric field ${ }^{13}$ which is helpful for eliminating hysteresis and improving response time but the operation voltage increases accordingly. Therefore, a delicate balance between hysteresis, response time and voltage is required in device design.

\section{CONCLUSION}

In conclusion, we investigated the dynamic relaxation of a polymer-stabilized BPLC composite and found that the response time becomes slower as the electric field exceeds the critical field, which is also the critical field for hysteresis to occur. The critical field is related to the Kerr constant of the BPLC material. We also proposed a possible mechanism to explain the relaxation behavior. These results will guide the future device design for keeping fast response time while suppressing hysteresis. ${ }^{21}$

\section{ACKNOWLEDGMENTS}

The authors are indebted to Industrial Technology Research Institute (Taiwan) for financial support.

${ }^{1}$ H. Kikuchi, M. Yokota, Y. Hisakado, H. Yang, and T. Kajiyama, Nature Mater. 1, 64 (2002).

${ }^{2}$ Y. Hisakado, H. Kikuchi, T. Ngamura, and T. Kajiyama, Adv. Mater. 17, 96 (2005).

${ }^{3}$ J. Yan, L. Rao, M. Jiao, Y. Li, H. C. Cheng, and S. T. Wu, J. Mater. Chem. 21, 7870 (2011).

${ }^{4}$ Z. Ge, S. Gauza, M. Jiao, H. Xianyu, and S. T. Wu, Appl. Phys. Lett. 94, 101104 (2009).

${ }^{5}$ Z. Ge, L. Rao, S. Gauza, and S. T. Wu, J. Disp. Technol. 5, 250 (2009).

${ }^{6}$ L. Rao, Z. Ge, S. T. Wu, and S. H. Lee, Appl. Phys. Lett. 95, 231101 (2009).

${ }^{7}$ M. Jiao, Y. Li, and S. T. Wu, Appl. Phys. Lett. 96, 011102 (2010).

${ }^{8}$ C. H. Lin, Y. Y. Wang, and C. W. Hsieh, Opt. Lett. 36, 502 (2011).

${ }^{9}$ J. Yan, Y. Li, and S. T. Wu, Opt. Lett. 36, 1404 (2011).

${ }^{10}$ K. M. Chen, S. Gauza, H. Xianyu, and S. T. Wu, J. Disp. Technol. 6, 49 (2010).

${ }^{11}$ Y. Chen, J. Yan, J. Sun, S. T. Wu, X. Liang, S. H. Liu, P. J. Hsieh, K. L. Cheng, and J. W. Shiu, Appl. Phys. Lett. 99, 201105 (2011).

${ }^{12}$ L. Rao, J. Yan, S. T. Wu, S. Yamamoto, and Y. Haseba, Appl. Phys. Lett. 98, 081109 (2011).

${ }^{13}$ H. C. Cheng, J. Yan, T. Ishinabe, and S. T. Wu, Appl. Phys. Lett. 98, 261102 (2011).

${ }^{14}$ A. Taugerbeck, P. Kirsch, D. Pauluth, J. Krause, J. Suermann, and M. Heckmeier, U.S. patent 7,223,150 B2 (29 May 2007).

${ }^{15}$ X. Zhu, Z. Ge, and S. T. Wu, J. Disp. Technol. 2, 2 (2006).

${ }^{16}$ J. Yan, H. C. Cheng, S. Gauza, Y. Li, M. Jiao, L. Rao, and S. T. Wu, Appl. Phys. Lett. 96, 071105 (2010).

${ }^{17}$ J. Yan, M. Jiao, L. Rao, and S. T. Wu, Opt. Express 18, 11450 (2010).

${ }^{18}$ H. C. Cheng, J. Yan, T. Ishinabe, N. Sugiura, C. Y. Liu, T. H. Huang, C. Y. Tsai, C. H. Lin, and S. T. Wu, J. Disp. Technol. 8, 98 (2012).

${ }^{19}$ E. Jakeman and E. P. Raynes, Phys. Lett. A 39, 69 (1972).

${ }^{20}$ H. Y. Wang, T. X. Wu, X. Y. Zhu, and S. T. Wu, J. Appl. Phys. 95, 5502 (2004).

${ }^{21}$ L. Rao, J. Yan, S. T. Wu, Y. C. Lai, Y. H. Chiu, H. Y. Chen, C. C. Liang, C. M. Wu, P. J. Hsieh, S. H. Liu, and K. L. Cheng, J. Disp. Technol. 7, 627 (2011). 